\title{
Photoluminescence in Tilted Magnetic Field of Triply Negatively Charged Excitons Hybridized with a Continuum
}

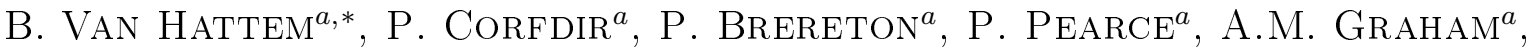 \\ M.J. Stanley ${ }^{a}$, M. Hugues ${ }^{b}$, M. Hopkinson $^{b}$ And R.T. Phillips ${ }^{a}$ \\ ${ }^{a}$ Cavendish Laboratory, University of Cambridge, J.J. Thomson Av., Cambridge CB3 0HE, United Kingdom \\ ${ }^{b}$ Department of Electronic and Electrical Engineering, University of Sheffield Mappin Str., Sheffield S1 3JD, United \\ Kingdom

\begin{abstract}
We analyse the magneto-photoluminescence of triply negatively charged excitons coupled to a continuum of states. The excitonic complex is confined to a Stranski-Krastanow InAs/GaAs quantum dot embedded in a Schottky diode. Different orientations of the magnetic field have been investigated. A modelling of the Coulomb blockade together with the calculation of the electron Fock-Darwin spectrum has allowed us to predict the magnetic fields of anticrossing between the quantum dot energy states and the wetting layer Landau levels. Good agreement between the theoretical model and the experimental results has been obtained.
\end{abstract}

DOI: 10.12693/APhysPolA.124.798

PACS: 78.66.Fd, 78.20.Ls

\section{Introduction}

Quantum dots in the Schottky diode structures can be sequentially charged until states that are hybridised with the continuum are occupied. Therefore they are a valuable tool for investigating the coupling between higher electronic states confined in the Stranski-Krastanow quantum dots (QDs) and the wetting layer [1-3]. While the photoluminescence (PL) energy of the neutral exciton $\left(\mathrm{X}^{0}\right)$, the trions and the doubly negatively charged exciton varies quadratically with the magnetic field, as a result of the Zeeman and diamagnetic effects [3], triply negatively charged excitons $\left(\mathrm{X}^{3-}\right)$ exhibit a much more complicated behaviour. As shown in Ref. [1], applying an external magnetic field first modifies the $\mathrm{X}^{3-}$ electronic configuration from open-shell (both $p$ shells are singly occupied) to closed-shell (one $p$ shell is doubly occupied). As a result, in the Faraday geometry and for magnetic field of about 1-2 T, the $\mathrm{X}^{3-}$ PL shows an abrupt redshift of the order of $\mathrm{meV}[1,3]$. Second, after recombination of the closed-shell $\mathrm{X}^{3-}$, the QD is left in an excited state, since its $s$ and $p$ shells are singly and doubly occupied, respectively. Relaxation occurs through an efficient Auger process that promotes one of the two $p$ electrons to the unbound QD $d$ shell [4]. The $d$ electron is coupled to the wetting layer continuum of states and in the presence of a magnetic field, the coherent interaction between the $d$ electron and the wetting layer manifests itself in the form of anticrossings [1, 3, 4].

In the present paper, we describe the evolution of the $\mathrm{X}^{3-} \mathrm{PL}$ for different orientations of the magnetic field with respect to the QD high symmetry axis. Then, based

*corresponding author; e-mail: bv252@cam.ac.uk on a modelling of the Coulomb blockade and on the calculation of the electron energy spectrum in the presence of a magnetic field, we predict the values of the anticrossing points between the $d$ state and the Landau levels.

\section{Sample and experimental setup}

The InAs/GaAs QD studied here is embedded in a Schottky diode that allows control of the charge state of the QD. The total active length of the diode and the distance between the QD plane and the back contact are $L_{\text {tot }}=225 \mathrm{~nm}$ and $L_{\text {back }}=25 \mathrm{~nm}$, respectively. The corresponding lever arm value that relates the applied gate voltage $V_{\mathrm{g}}$ to the $\mathrm{QD}$ potential $\phi_{\mathrm{QD}}$ is $\lambda=e\left(V_{0}-V_{\mathrm{g}}\right) / \Phi_{\mathrm{QD}}=L_{\mathrm{tot}} / L_{\mathrm{back}}=9$, where $V_{0}$ is the Schottky barrier height. Magneto-photoluminescence experiments have been carried out at $4.5 \mathrm{~K}$ in a confocal setup $[3,5]$. The QD was excited non-resonantly using a continuous wave $\mathrm{Ti}: \mathrm{Al}_{2} \mathrm{O}_{3}$ laser tuned to $780 \mathrm{~nm}$. The QD sample and the confocal objective are mounted on a support that can rotate with respect to the applied magnetic field. Magnetic fields up to $10 \mathrm{~T}$ have been obtained with a superconducting magnet cooled down to $5 \mathrm{~K}$. In this work, the angle $\theta$ between the sample and the magnetic field has been set to 0,45 , and $90^{\circ}$.

\section{Results}

We show in Fig. 1a the QD PL at zero magnetic field for $V_{\mathrm{g}}$ between -0.8 and $-0.2 \mathrm{~V}$. Charging events at $V_{\mathrm{g}}=-0.68$ and $-0.40 \mathrm{~V}$ result in abrupt shifts in the QD PL energy. Following the procedure described in Ref. [6], we attribute the charging plateau at $V_{\mathrm{g}}>-0.4$ to the negative trion $\mathrm{X}^{-}$. Accordingly, the $\mathrm{X}^{0}$ and the positive trion $\mathrm{X}^{+}$occur for $-0.68<V_{\mathrm{g}}<-0.4 \mathrm{~V}$ and $V_{\mathrm{g}}<-0.8 \mathrm{~V}$, respectively. Using the Coulomb model described in Ref. [7], it is possible to extract from the bias 


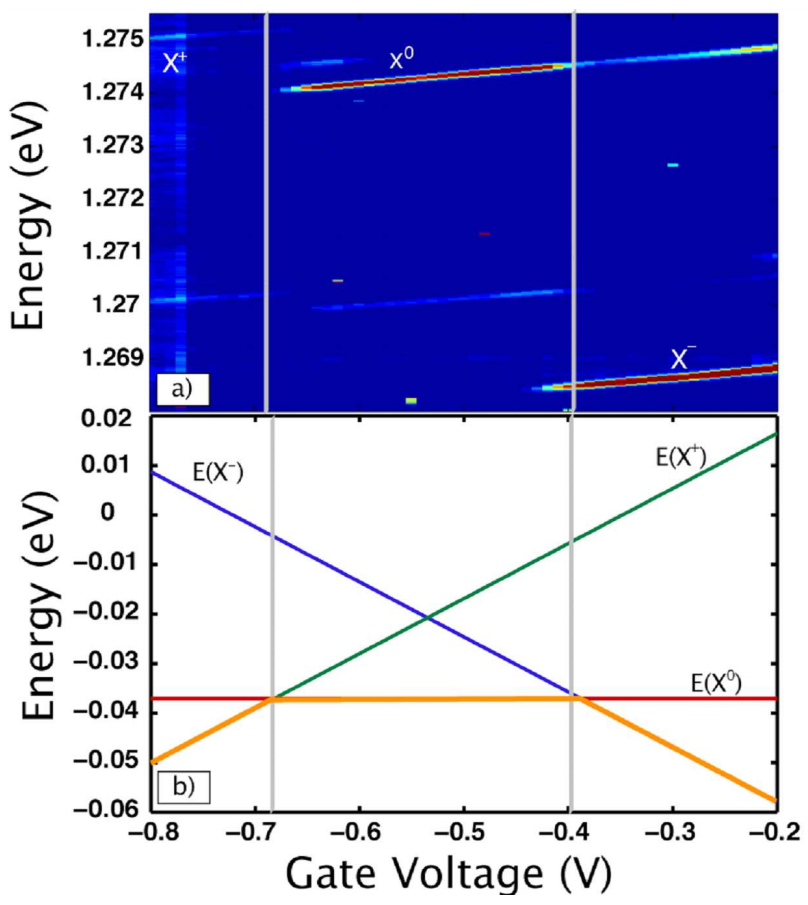

Fig. 1. (a) Photoluminescence at zero magnetic field with respect to the applied gate voltage. The vertical solid lines show the charging transitions from the positive to the negative trion. (b) Gate voltage dependence of the energy of the positive trion $\mathrm{E}\left(\mathrm{X}^{+}\right)$, of the neutral exciton $\mathrm{E}\left(\mathrm{X}^{0}\right)$ and of the negative trion $\mathrm{E}\left(\mathrm{X}^{-}\right)$(green, red and blue lines, respectively). The orange line indicates the lowest energy charge state. map in Fig. 1a the repulsive Coulomb energy between two $s$ electrons in the QD $\left(E_{\mathrm{e}}^{s s}\right)$ and the electron in-plane quantization energy $\hbar \omega_{\mathrm{e}, \perp}$. As shown in Fig. 1b, we deduce $E_{\mathrm{e}}^{s s}=30.3 \mathrm{meV}$ and $\hbar \omega_{\mathrm{e}, \perp}=50 \mathrm{meV}$, which are typical values for strongly confined InAs QDs [7]. Since the QD studied is lens-shaped [8], we assume that the electron on-axis quantization energy is $\hbar \omega_{\mathrm{e}, z}=250 \mathrm{meV}$. Using an electron mass $m_{\mathrm{e}}=0.08 m_{0}$ and the quantization energies obtained above, it is possible to calculate the electron energy spectrum in the presence of a magnetic field. We have computed the eigenvalues of the Fock-Darwin Hamiltonian for an electron in a QD in the presence of a tilted magnetic field using the analytical formula provided in Ref. [9]. Note that our modelling only accounts for orbital Zeeman effects. The results of our calculations for $\theta=0,45$, and $90^{\circ}$ are displayed in Fig. 2d-f.

The $\mathrm{X}^{3-}$ magneto-PL spectra are displayed in Fig. $2 \mathrm{a}-\mathrm{c}$ for $\theta=0,45$, and $90^{\circ}$. We attribute the emission lines centred at 1266.5 and $1267.9 \mathrm{meV}$ to the recombination of the closed-shell and open-shell $\mathrm{X}^{3-}$, respectively. The $0.79 \mathrm{meV}$ broadening observed for the closed-shell $\mathrm{X}^{3-} \mathrm{PL}$ arises from the efficient Auger-mediated reconfiguration of the final state transition [4]. In the Faraday geometry, when increasing the magnetic field, the emission from the open-shell $\mathrm{X}^{3-}$ quenches to the benefit of that from the closed-shell $\mathrm{X}^{3-}$. In agreement with the discussion in Refs. [1, 3] the larger the magnetic field, the larger the energy splitting between the two $p$ shells and therefore the more favoured the closed-shell configuration.
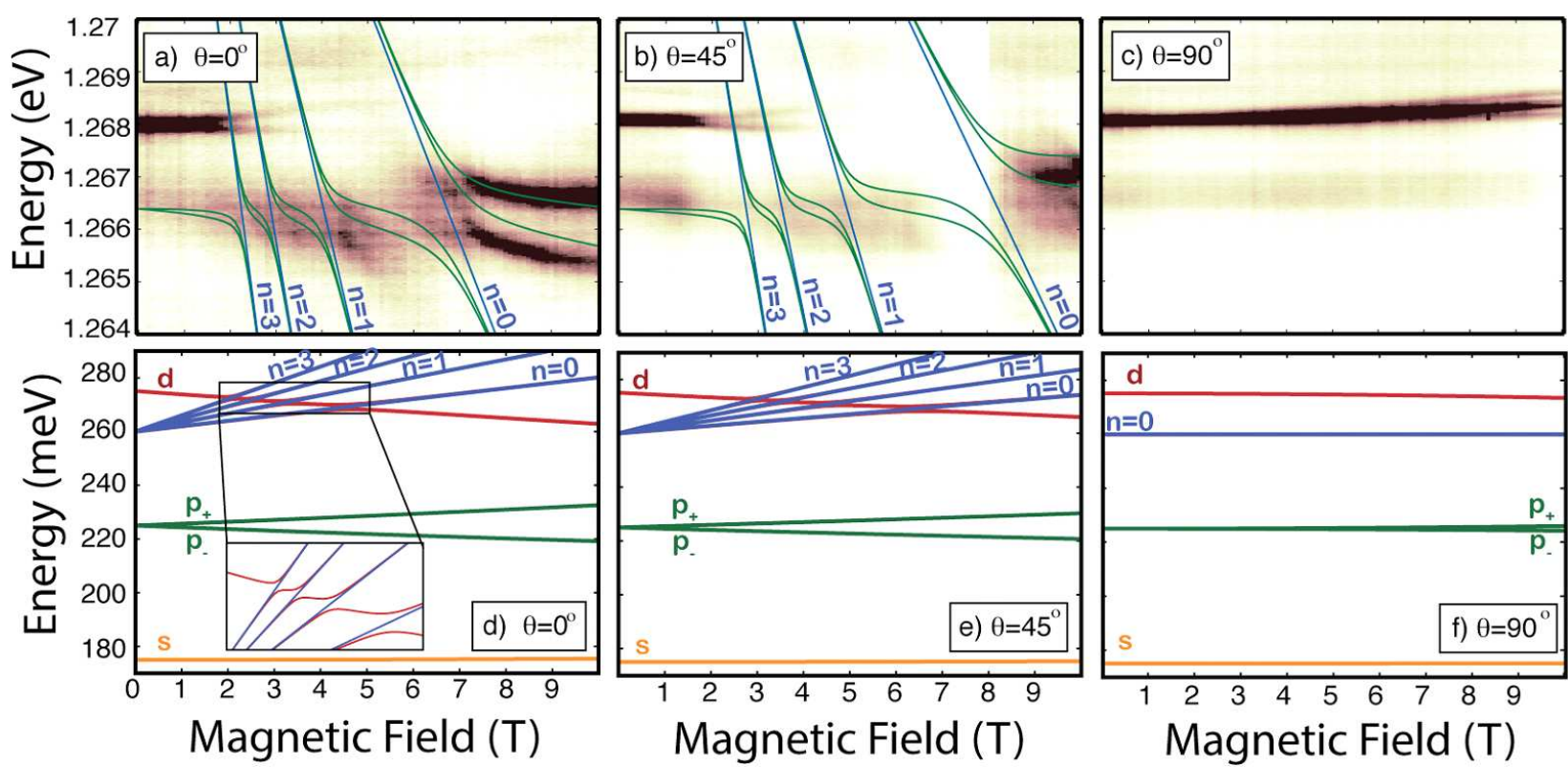

Fig. 2. (a-c) Magneto-photoluminescence of the $\mathrm{X}^{3-}$ for $\theta=0^{\circ}$ (a), $45^{\circ}$ (b), and $90^{\circ}$ (c). PL lines are not polarisation-resolved. Blue and green lines are guides to the eye indicating the anticrossing behaviour and the Landau levels' energies. (d-f) Calculated $s, p$, and $d$ quantum dot energy levels (orange, green and red lines, respectively) and wetting layer Landau levels (blue) for $\theta=0^{\circ}(\mathrm{d}), 45^{\circ}(\mathrm{e})$, and $90^{\circ}(\mathrm{f})$. The inset in (d) highlights the anticrossings between the $d$ energy level and the Landau levels. 
We also remark that the magnetic field range where the open-shell $\mathrm{X}^{3-}$ PL dominates over the closed-shell emission increases with $\theta$. When $\theta=90^{\circ}$, the PL from the open-shell $\mathrm{X}^{3-}$ even dominates the spectrum for the whole range of investigated magnetic field. We attribute this to the fact that the energy splitting between the two $p$ states is directly proportional to $B \cos \theta$. We expect the closed-shell $\mathrm{X}^{3-} \mathrm{PL}$ to exhibit anticrossings when the energy of the QD $d$ shell corresponds to the energy of the wetting layer Landau levels. We previously reported that at zero magnetic field, the energy separation between the QD $d$ shell and the $\Gamma$ point of the wetting layer conduction band is $\Delta=15 \mathrm{meV}$ [3]. The magnetic field dependence of the $n$-th Landau level energy is therefore given by $E_{d}(0)-\Delta+(n+3 / 2) e \hbar B / m_{\mathrm{e}}$, where $E_{d}(0)$ is the QD $d$ orbital energy at zero magnetic field.

We plot in Fig. 2d-f the magnetic field dependence of the energy of the first four Landau levels. For $\theta=0^{\circ}$, we expect to observe anticrossings for $B=2,2.5,3.25$, and $4.75 \mathrm{~T}$ (Fig. 2d). Experimentally, the anticrossings arising from the hybridization of the $d$ level with the $n=0$ and $n=1$ Landau levels occur for $B=3.75$ and $5.9 \mathrm{~T}$ (Fig. 2a). We believe that the discrepancy between our modelling and the data originates mainly from the crude approximation made on the value of $\hbar \omega_{\mathrm{e}, z}$. The same comment goes for $\theta=45^{\circ}$, where only qualitative agreement is reached between the experimental data and our calculations (Fig. 2b,e). Finally, in the Voigt geometry, the closed-shell $\mathrm{X}^{3-}$ PL shows no anticrossing, as expected from our simulations.

\section{Conclusion}

We have studied the photoluminescence in magnetic field of $\mathrm{X}^{3-}$ complexes confined to InAs/GaAs Stranski-Krastanow quantum dots. The $\mathrm{X}^{3-}$ magneto-photoluminescence shows several anticrossings that result from the coupling between the final state of the $\mathrm{X}^{3-}$ transition and the wetting layer. After extraction of the electron quantisation energies from the photoluminescence bias map, we have calculated the electron Fock-
Darwin spectrum. We could then predict the magnetic field of anticrossing between the wetting layer Landau levels and the quantum dot $d$ state. We obtained a good agreement between the calculated electronic energies dispersion and the experimental data for all the orientations of the magnetic field.

\section{Acknowledgments}

We acknowledge funding from the EPSRC, the Hitachi Cambridge Laboratory and from the European Union Seventh Framework Program (grant No. 265073).

\section{References}

[1] K. Karrai, R.J. Warburton, C. Schulhauser, A. Högele, B. Urbaszek, E.J. McGhee, A.O. Govorov, J.M. Garcia, B.D. Gerardot, P.M. Petroff, Nature 427, 135 (2004).

[2] N.A.J. Kleemans, J. van Bree, A.O. Govorov, J.G. Keizer, G.J. Hamhuis, R. Nötzel, A.Yu. Silov, P.M. Koenraad, Nature Phys. 6, 534 (2010).

[3] B. Van Hattem, P. Corfdir, P. Brereton, P. Pearce, A.M. Graham, M.J. Stanley, M. Hugues, M. Hopkinson, R.T. Phillips, Phys. Rev. B 87, 205308 (2013).

[4] A. Govorov, K. Karrai, R. Warburton, A. Kalameitsev, Physica E 20, 295 (2004).

[5] T. Kehoe, M. Ediger, R.T. Phillips, M. Hopkinson, Rev. Sci. Instrum. 81, 013906 (2010).

[6] M. Ediger, G. Bester, A. Badolato, P. Petroff, K. Karrai, A. Zunger, R. Warburton, Nature Phys. 3, 774 (2007).

[7] R.J. Warburton, B.T. Miller, C.S. Dürr, C. Bödefeld, K. Karrai, J.P. Kotthaus, Phys. Rev. B 54, 16221 (1998).

[8] The lens-shaped symmetry of the quantum dot has been deduced from the $\theta$ dependence of the $\mathrm{X}^{0}$ diamagnetic coefficient, see Ref. [3].

[9] A.B. Henriques, A.L. Efros, Phys. Rev. B 80, 153302 (2009). 